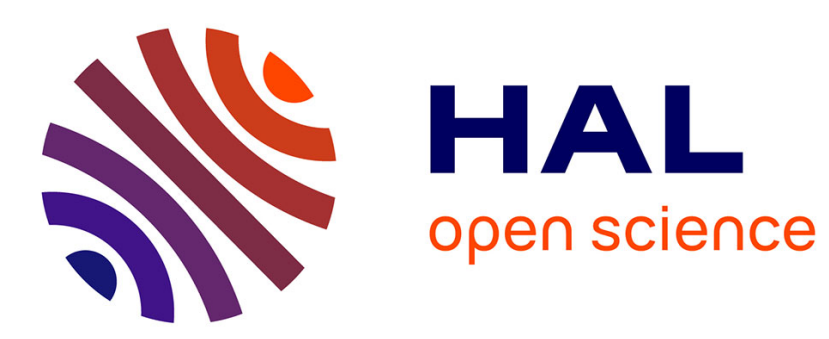

\title{
Enhanced Biological Activity of BMP-2 Bound to Surface-Grafted Heparan Sulfate
}

\author{
Elisa Migliorini
}

\section{To cite this version:}

Elisa Migliorini. Enhanced Biological Activity of BMP-2 Bound to Surface-Grafted Heparan Sulfate. Advanced Biosystems, 2017, 1 (4), pp.1600041. 10.1002/adbi.201600041 . hal-02324796

\section{HAL Id: hal-02324796 \\ https://hal.science/hal-02324796}

Submitted on 9 Sep 2020

HAL is a multi-disciplinary open access archive for the deposit and dissemination of scientific research documents, whether they are published or not. The documents may come from teaching and research institutions in France or abroad, or from public or private research centers.
L'archive ouverte pluridisciplinaire HAL, est destinée au dépôt et à la diffusion de documents scientifiques de niveau recherche, publiés ou non, émanant des établissements d'enseignement et de recherche français ou étrangers, des laboratoires publics ou privés. 


\title{
Enhanced Biological Activity of BMP-2 Bound to Surface-Grafted Heparan Sulfate
}

\author{
Elisa Migliorini, * Patrick Horn, Tamás Haraszti, Seraphine V. Wegner, Christian Hiepen, \\ Petra Knaus, Ralf P. Richter, and Elisabetta Ada Cavalcanti-Adam*
}

Over the last decade, there has been a growing interest in the development of new materials to improve bone morphogenetic protein-2 (BMP-2) delivery for tissue regeneration. This study reports the development and application of model surfaces that present BMP-2 via heparan sulfate (HS), a ubiquitous component of the extracellular matrix (ECM). On these surfaces, HS is grafted by its reducing end, to mimic the natural arrangement of HS proteoglycans in the ECM. The binding of each component on these biomimetic surfaces is highly controlled, in terms of stoichiometry of molecules and BMP-2/ grafted-HS affinity, as determined by surface-sensitive techniques. For comparison, this study also uses surfaces presenting immobilized BMP-2 alone. Functional validations of the surfaces are performed using a murine myoblast cell line ( $\mathrm{C} 2 \mathrm{C} 12)$ and primary human mesenchymal stromal cells. In both cell types, HS-bound BMP-2 and surface-immobilized BMP-2 significantly prolong SMAD 1/5 phosphorylation, compared to BMP-2 added to the culture media. Moreover, HS-bound BMP- 2 enhances p-SMAD 1/5 levels in C 2 C 12 cells and reduces noggin antagonistic activity. Thus, grafted HS positively affects BMP-2 cellular activity. This innovative surface design, which mimics natural interactions of growth factors with ECM components, constitutes a promising candidate for future regenerative medicine applications.

Bone morphogenetic protein-2 (BMP-2) is known to initiate the differentiation of mesenchymal stem cells (MSCs) into osteoblasts and chondrocytes in vivo and in vitro, ${ }^{[1]}$ as well as the transdifferentiation of mouse myoblasts C2C12 cell line into mineralizing bonelike cells. ${ }^{[2,3]}$ The BMP-2 signaling pathway is activated by the binding of the growth factor to two types of transmembrane serine/threonine kinase receptors, the BMP type I (BMPRI) and type II (BMPRII) receptors. Binding of BMP-2 to BMPRI and BMPRII induces the phosphorylation of the SMAD1/5/8 complex, which then together with co-SMAD (SMAD4) translocates to the nucleus and, with other DNA-binding proteins, participates in the transcriptional regulation of osteogenic target genes. ${ }^{[4]}$

The clinical use of BMP-2 was approved in 2002 by the Food and Drug Administration in the USA and validated by the national Medical Agencies in Europe. BMP-2 has been rapidly introduced into orthopedic clinical practice ${ }^{[5]}$ but, in some cases, even high doses (up to $2 \mathrm{mg}$ per level) appear only marginally effective, likely due to an autocrine BMP-2 inhibition by noggin the major extracellular BMP antagonist, at sites of BMP-2 application. ${ }^{[6]}$ Therefore, improving BMP-2 activity, by using delivery systems that suppress BMP-2 inhibition by noggin, would help in overcoming current challenges in BMP-2 clinical application. ${ }^{[7]}$
Dr. E. Migliorini, Dr. S. V. Wegner, Prof. E. A. Cavalcanti-Adam

Department of Biophysical Chemistry

Institute of Physical Chemistry

Heidelberg University

Im Neuenheimer Feld 253, 69120 Heidelberg, Germany

E-mail:migliorini@is.mpg.de;

ada.cavalcanti-adam@urz.uni-heidelberg.de

Dr. E. Migliorini, Dr. S. V. Wegner, Prof. E. A. Cavalcanti-Adam Department of Cellular Biophysics

Max Planck Institute for Medical Research

Heisenbergstr. 3, D-70569 Stuttgart, Germany

Dr. P. Horn

Department of Medicine V

Heidelberg University

INF 410, 69120 Heidelberg, Germany

Dr. T. Haraszti

DWI - Leibniz Institute for Interactive Materials

Forkenbeckstr. 50, 52056 Aachen, Germany

DOI: 10.1002/adbi.201600041

\author{
Dr. S. V. Wegner \\ Max Planck Institute for Polymer Research \\ Ackermannweg 10, 55128 Mainz, Germany \\ Dr. C. Hiepen, Prof. P. Knaus \\ Institute of Biochemistry \\ Freie Universität Berlin \\ Thielallee 63, 14195 Berlin, Germany \\ Dr. R. P. Richter \\ School of Biomedical Sciences and School \\ of Physics and Astronomy \\ University of Leeds \\ Leeds LS2 9JT, UK \\ Dr. R. P. Richter \\ Biosurfaces Lab \\ CIC biomaGUNE \\ Paseo Miramon 182, 20009 San Sebastian, Spain \\ Dr. R. P. Richter \\ Laboratory of Interdisciplinary Physics \\ University Grenoble-Alpes and CNRS \\ 140 Rue de la Physique, 38402 St. Martin d'Hères, France
}



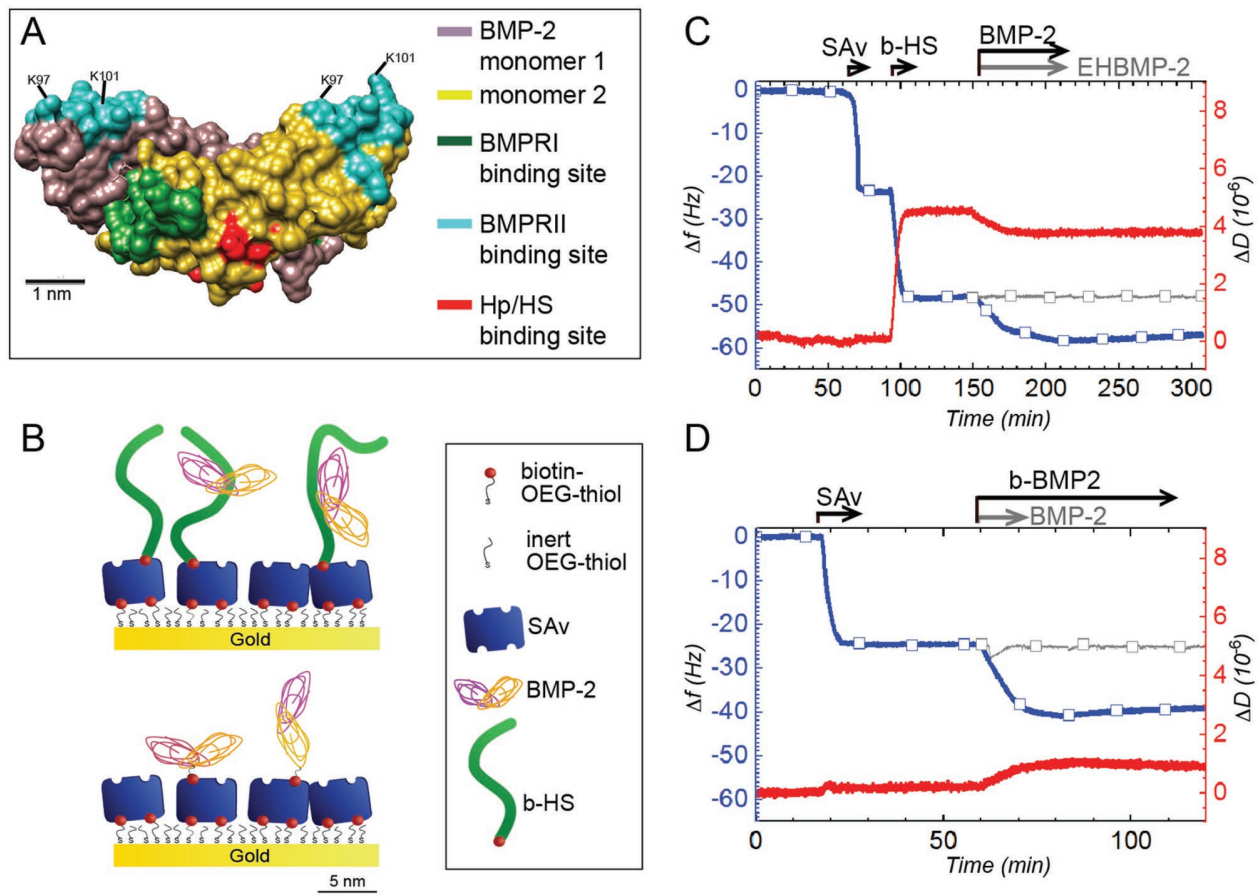

$\mathrm{D}$

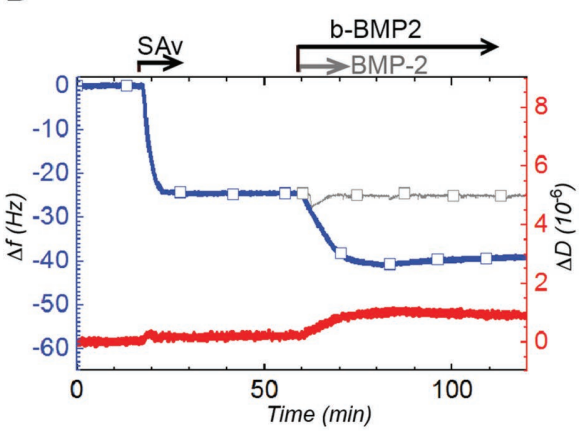

Figure 1. A) BMP-2 homodimer structure (PDB: 3BMP). B) Schematic representation of the biomimetic platforms. C,D) QCM-D characterization. Frequency shifts: $\Delta f$-blue lines with square symbols; dissipation shifts: $\Delta D$-red lines. Start and duration of incubation steps are indicated by arrows; during all other times, the surface was exposed to working buffer.

The development of materials that are able to control BMP-2 molecular presentation and local concentration is an essential approach for a deeper understanding of BMP-2 functions and the modulation of its biological activity. ${ }^{[8]}$

In tissues, BMP-2 is bound to extracellular matrix (ECM) components such as proteins ${ }^{[9]}$ and glycosaminoglycans, in particular to heparan sulfate (HS). ${ }^{[10,11]}$ BMP-2 is a homodimeric protein and has been reported to bind in vitro to heparin ( $\mathrm{Hp})$, a highly sulfated form of HS, via a binding site located at the N-terminus (Figure 1A) with an affinity of $\approx 20 \times 10^{-9} \mathrm{M}^{[12]}$ The binding of BMP-2 to HS, on the other hand, remains to be fully characterized in terms of stoichiometry, affinity, and concomitant HS morphological changes.

HS, a linear polysaccharide formed by variably sulfated repeating disaccharide units, is typically covalently attached to core proteins forming HS proteoglycans (HSPGs). The functions of HSPGs depend on their structure and location, being either at the cell surface or as part of the ECM. ${ }^{[13]}$

Contradictory effects of cell surface HSPGs on BMP-2 activity have been reported. On the one hand, endogenous HSPGs negatively modulate both chondrogenic ${ }^{[14]}$ and osteogenic $^{[10]}$ differentiation by inhibiting the activation of BMP-2 signaling. On the other hand, HSPGs act as BMP-2 coreceptors, promoting the formation of complexes between BMPRII and BMPRI, thereby enhancing the bioactivity of BMP-2. ${ }^{[15]}$ Interestingly, the BMP antagonist noggin also binds to HS.[16] Once bound to cell surface HSPGs, noggin maintains its function to inhibit the activity of BMP-4, another member of the BMP family, by blocking the binding epitopes for BMPRI and BMPRII. ${ }^{[16,17]}$
To date, the role of HSPGs in the extracellular space (ECMHSPGs) on BMP-2 bioactivity is only partially explored. The main functions so far attributed to ECM-HSPGs are to prevent the diffusion of growth factors away from the regions where they are likely to be required. ${ }^{[18]}$ However, it is still unknown if these HSPGs can positively modulate BMP-2 biological activity thus enhancing in vitro osteogenic differentiation. ${ }^{[19]}$

The presence of HS added to the culture media of BMP. responsive cells (C2C12 myoblasts) prolongs SMAD 1/5/8 phosphorylation in these cells and reduces the interactions with the antagonist noggin, ${ }^{[11,20]}$ in contrast to the effect of cell surface HSPGs. ${ }^{[16]}$ Since in vivo HS is not in solution, but rather covalently bound to core proteins through its reducing end forming HSPGs ${ }^{[21]}$ administering HS in the cell culture media is unlikely to be representative of the in vivo extracellular HS.

Biomimetic platforms presenting $\mathrm{HS}$ in a bound and oriented manner are therefore suitable to explore (i) the characteristics of HS/BMP-2 binding at the molecular and cellular levels, (ii) the impact of BMP-2 presentation on BMP-2 activity, and (iii) the interaction of noggin with grafted HS and its recognition of the HS-bound BMP-2. The strength of such platforms lies in the precise molecular presentation, which gives the ability to control and characterize BMP-2 interactions with HS, as well as the possibility to further exploit them as substrates for investigations on the impact of HS on BMP-2 activity in cells. We designed our model surfaces in a way that HS is grafted by its reducing end, ${ }^{[22]}$ mimicking its attachment to HSPG core proteins in vivo. To this end, we functionalized the reducing end of HS with biotin and used gold-coated surfaces functionalized first with a mixed monolayer of oligoethyleneglycol (OEG)-thiol 
and biotin-OEG-thiol and then with a streptavidin (SAv) monolayer as substrates ${ }^{[23]}$ (Figure 1B). The OEG monolayer passivates against nonspecific binding of proteins, here tested using bovine serum albumin (Figure S1, Supporting Information), whereas $S A v$ binds through a specific interaction with the biotin end groups and forms a rigid monolayer. Such monolayer serves then as a mediator for the immobilization of biotinylated compounds such as biotinylated HS (b-HS; Figure 1C) and biotinylated BMP-2 (b-BMP2; Figure 1D). BMP-2 binds to $\mathrm{SAv}$ via a biotin- $\mathrm{PEG}_{12}-\mathrm{NHS}$ (PEG, poly(ethylene glycol); NHS, $N$-hydroxysuccinimide) ester linker (of $\approx 5.6 \mathrm{~nm}$ contour length), which reacts with primary amines of the growth factor. Surface functionalization steps, specificity of the molecular binding and mechanical properties of the biomolecular films were characterized by quartz crystal microbalance with dissipation monitoring (QCM-D); adsorption and desorption rates of the different biomolecules, as well as biomolecular surface densities, were quantified by spectroscopic ellipsometry (SE).

Figure 1C,D shows QCM-D characterization of the two biomimetic platforms ( $\Delta f$ and $\Delta D$ correspond to the fifth overtone). After the formation of a SAv monolayer with an expected thickness of $\approx 4 \mathrm{~nm}^{[24]}$ (determined using Sauerbrey's equation; Figure 1C, 60 to $80 \mathrm{~min}$ ), b-HS adsorbs rapidly forming a hydrated and soft film, indicated by the increase in dissipation of $4.5 \pm 0.5 \times 10^{-6}$ (Figure 1C, 90-110 min). BMP-2, incubated at a concentration of $96 \times 10^{-9} \mathrm{M}$, stably binds to the b-HS film causing an increase in film rigidity, as demonstrated by the negative dissipation shift of $-0.9 \pm 0.1 \times 10^{-6}$ (Figure 1C, 150-180 min). Complementary assays based on fluorescence recovery after photobleaching, using films of b-HS grafted to supported lipid bilayers in the fluid phase, revealed that BMP-2 binding reduces the lateral mobility of HS chains substantially. This suggests that the increased rigidity arises from crosslinking of HS chains mediated by the growth factor (Figure S2, Supporting Information). This phenomenon has been reported for other ECM signaling proteins, such as chemokines and growth factors, some of which present multiple HS binding sites. ${ }^{[25]}$ We speculate that HS cross-linking induced by BMP-2 might be due to the presence of two or more independent $\mathrm{Hp} /$ HS binding sites at the N-terminus region of the BMP-2 dimer, as previously predicted. ${ }^{[26]} \mathrm{A}$ mutant form of BMP-2, EHBMP-2, where the N-terminal region responsible for the Hp binding was substituted by a heterologous sequence from human interleukin-2, ${ }^{[12]}$ does not bind to b-HS (Figure 1C, gray curve) and does not induce HS film cross-linking (Figure S2, Supporting Information). This demonstrates that BMP-2 binds HS specifically through the same site as Hp.

For comparison, we also adopted a second BMP-2 immobilization strategy that does not involve HS. In this case, biotinylated BMP-2 (b-BMP2) binds directly to the SAv monolayer until saturation (Figure 1D, 50-110 min). When incubated at the same concentration $\left(96 \times 10^{-9} \mathrm{M}\right)$ without the biotin tag, BMP-2 does not bind stably (Figure 1D, gray curve) demonstrating the b-BMP2 is specifically attached via biotin to the SAv monolayer.

To quantify the binding strength of BMP-2 to b-HS films, we used SE and performed a titration assay (Figure S3A, Supporting Information). The titration curve (Figure S3B, Supporting Information) was approximated well by the simple Langmuir isotherm
$\Gamma_{\text {eq }}=\Gamma_{\text {max }} \frac{[\mathrm{BMP}-2]}{K_{\mathrm{d}}[\mathrm{BMP}-2]}$

with an affinity of $K_{\mathrm{d}} \approx 1.6 \mu \mathrm{m}$ and a maximal BMP-2 surface density of $\Gamma_{\max } \approx 1000 \mathrm{ng} \mathrm{cm}^{-2}$. For this analysis, it has to be considered that HS is not a homogeneous polymer: the constituent monosaccharides are variably sulfated and/or might exist as different epimers, (regions of high sulfation coexisting with regions of low sulfation along individual HS chains). These structural features can also vary from one HS source to another. ${ }^{[27,28]}$ It is therefore likely that HS presents a spectrum of binding sites rather than a single type. The affinity constant of $1.6 \mu \mathrm{m}$ should thus be considered as an effective value, resulting from a spectrum of binding sites with different affinities. ${ }^{[29]}$ For comparison, an affinity of $20 \times 10^{-9}$ M for Hp has been previously reported ${ }^{[12]}$ indicating that a high degree of sulfation might indeed substantially enhance the effective binding strength in comparison to the value obtained here.

At the maximal surface density predicted by the Langmuir isotherm $\left(\Gamma_{\max }=1000 \mathrm{ng} \mathrm{cm}^{-2}\right)$, we calculated that up to 11 BMP-2 dimers would bind to a $12 \mathrm{kDa}$ b-HS chain; in such condition $\approx 2.1$ HS disaccharides are available on average per BMP-2 dimer. A small nonspecific binding of BMP-2 to the SAv monolayer has to be considered when high BMP-2 concentrations are used (Figure S4A, Supporting Information). At high concentration, it is also possible that several BMP-2 molecules interact with each other forming aggregates (Figure S4B, Supporting Information). Moreover, BMP-2 in solution has been found to have a limited physical stability, with aggregates of various sizes forming in a $\mathrm{pH}$-dependent manner. ${ }^{[30]}$ Future studies using shorter oligosaccharides (down to 3 disaccharides) would be useful to study the minimal HS/Hp disaccharides length able to bind BMP-2.

The desorption of BMP-2 upon buffer rinsing is well described by the exponential function (Figure S3A, Supporting Information, red line)

$\Gamma=\Gamma_{\mathrm{r}} e^{-k_{\mathrm{off}} \Delta t}+\Gamma_{\mathrm{ir}}$

with an apparent off-rate $k_{\text {off }}=6.1 \pm 1.9 \times 10^{-4} \mathrm{~s}^{-1} . \Gamma_{\text {ir }}$ and $\Gamma_{\mathrm{r}}$ correspond, respectively, to BMP-2 fractions that are irreversibly and reversibly bound to the b-HS film. The fit reveals similar values for $\Gamma_{\mathrm{ir}}$ and $\Gamma_{\mathrm{r}}$, meaning that $\approx 50 \%$ of BMP- 2 is released from the b-HS film. The heterogeneous structure of HS chains ${ }^{[28]}$ and the spectrum of binding sites and affinities that results from it may well explain the presence of a BMP-2 fraction that binds HS reversibly and another fraction that binds HS stably.

SE was also used to control the surface density of the active biomolecules, a fundamental piece of information to perform studies on BMP-mediated cellular responses (Figure 2). To this end, the assembly of biomimetic surfaces was followed step by step, as for the QCM-D measurements. The areal mass densities obtained from the SE data are reported in Table 1.

b-HS binds to SAv (Figure 2A and Table 1) and, considering SAv molecular mass of $60 \mathrm{kDa}$, the amount of HS bound on average per available biotin-binding site (assuming that two of four sites engage in the immobilization to the surface) is $6 \pm$ $1.6 \mathrm{kDa}$. This value is below the average HS molecular mass 
A

SAv b-HS

BMP-2

noggin

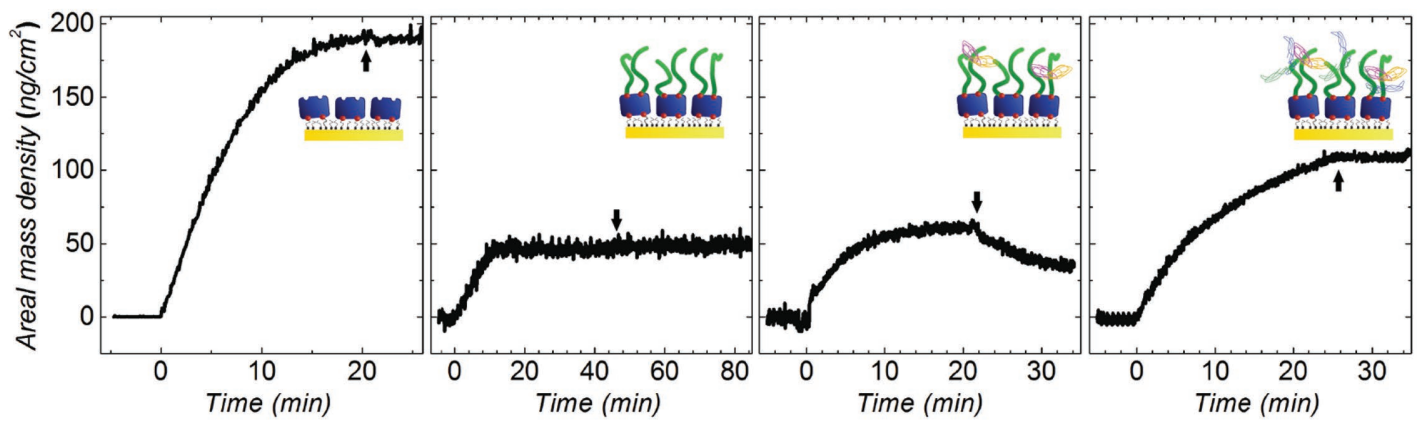

$\mathrm{B}$

b-BMP2

noggin

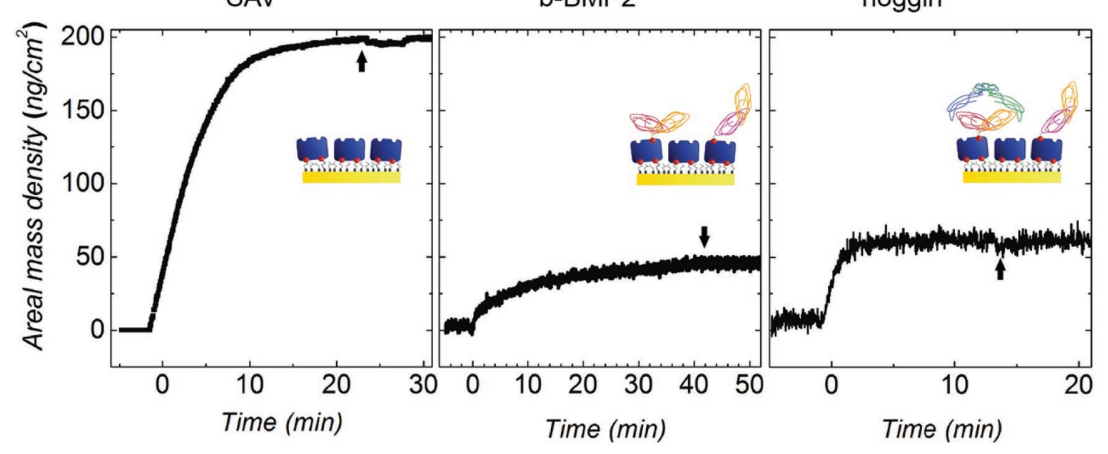

Figure 2. Surface functionalization is followed in situ by SE on a gold-supported OEG monolayer. A,B) Sample concentrations are: $83 \times 10^{-9} \mathrm{M}$ SAv, $0.8 \times 10^{-6} \mathrm{M}$ b-HS, $96 \times 10^{-9} \mathrm{M}$ BMP-2, b-BMP2, and $200 \times 10^{-9} \mathrm{M}$ noggin. Each incubation step started at 0 min; start of rinsing in working buffer is indicated by arrows.

employed (12 kDa). As previously discussed, ${ }^{[23]}$ this discrepancy is likely to be due to the large size distribution of HS in solution, i.e., capture on SAv has selected the shorter chains in the initial HS sample.

Thanks to the quantification of surface densities afforded by SE, we estimate that, at $96 \times 10^{-9}$ M concentration, each BMP-2 dimer has $\approx 20 \mathrm{kDa}$ of $\mathrm{HS}$, corresponding to roughly 36 disaccharides, available on average at signal stabilization.

Noggin, does not bind to SAv (Figure S5A, Supporting Information), as expected, but has a HS/Hp binding site ${ }^{[31]}$ and indeed readily binds to the native $\mathrm{b}$-HS film, at an areal mass density of

Table 1. Data were extracted from SE measurements at signal stabilization (Figure 2). Mean values and standard errors from the mean were derived from three independent measurements. On the basis of these numbers, we quantify the number of molecules grafted per unit surface area and the stoichiometry of binding.

\begin{tabular}{lc}
\hline Compound & Areal mass density $\left[\mathrm{ng} \mathrm{cm}^{-2}\right]$ \\
\hline SAv & $204.3 \pm 9.9$ \\
+ b-BMP2 & $44.2 \pm 4.6$ \\
+ noggin & $57.6 \pm 2.1$ \\
b-HS & $40.7 \pm 3.5$ \\
+ BMP-2 (eq) & $53.2 \pm 4.6$ \\
+ BMP-2 (rins) & $35.3 \pm 2.7$ \\
+ Noggin & $112 \pm 4.1$ \\
\hline
\end{tabular}

(eq) value close to equilibrium during BMP-2 injection; (rins) Value after BMP-2 rinsing with working buffer until plateau was reached. $\approx 112 \pm 0.7 \mathrm{ng} \mathrm{cm}^{-2}$ when incubated at $\approx 200 \times 10^{-9} \mathrm{M}$ (Figure S5B, Supporting Information). When BMP-2 loaded b-HS film is incubated with noggin at the same concentration, noggin binds to the film (Figure 2A and Table 1). The total amount of b-HS bound proteins (BMP-2 + noggin) is higher than the amount the individual proteins adsorbed on b-HS. This indicates that both proteins can be present in the b-HS film simultaneously, however it remains unclear whether b-HS-bound noggin recognizes at the same time BMP-2.

At a concentration of $96 \times 10^{-9} \mathrm{M}$, one b-BMP2 binds on average to two SAv molecules (Figure 2B), which is expected to be due to the larger dimensions of BMP-2 compared to SAv $(\approx 8 \mathrm{~nm}$ vs $\approx 5 \mathrm{~nm})$. After immobilization of b-BMP2 on SAv, we added noggin and observed that it binds at a ratio of $1 \mathrm{~b}-\mathrm{BMP} 2$ to 0.7 noggin molecules (Figure 2A). As noggin is known to bind BMPs with a $1: 1$ stoichiometry, ${ }^{[17]}$ this implies that $\approx 30 \%$ of immobilized b-BMP2 is apparently not recognized by noggin. Plausible explanations are that the noggin binding site of some BMP-2 molecules is oriented toward the surface and therefore not accessible, and/or that the biotin-PEG-NHS is reacting with Lys 97 and/or Lys 101, thus protecting the binding epitope recognised by noggin ${ }^{[7]}$ (Figure 1A). Noggin inhibits BMP signaling by blocking BMP binding epitopes for both BMPRI and BMPRII. ${ }^{[17]}$ It is therefore likely that all b-BMP2 molecules recognized by noggin are also accessible to cell surface receptors.

To understand how the type of BMP-2 immobilization impacts on BMP-2 and noggin biological activity, we next performed functional experiments using BMP-responsive cells. Murine C2C12 myoblasts and mesenchymal stem cells from human bone 
A

C2C12 cells
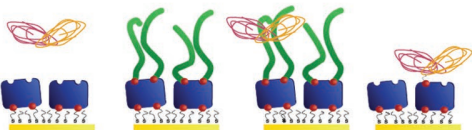

3090180309018030901803090180 min
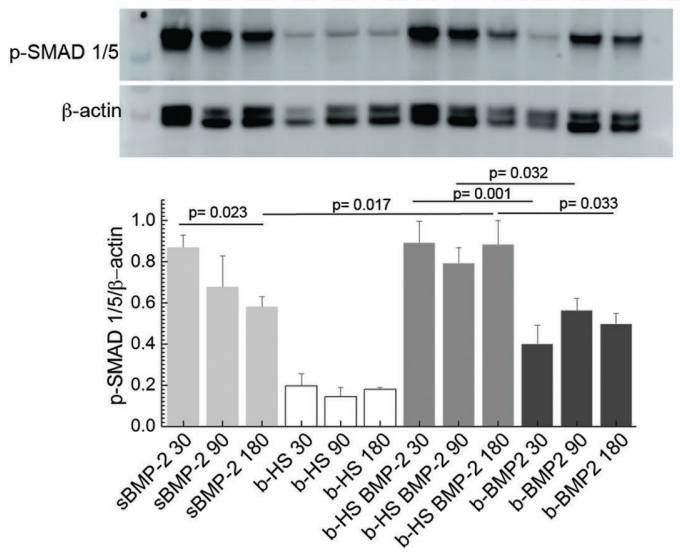

C

\section{hMSCs}

sBMP-2 b-HS b-HS BMP-2 b-BMP2

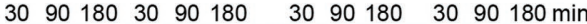

B

B $2 \mathrm{C} 12$ cells
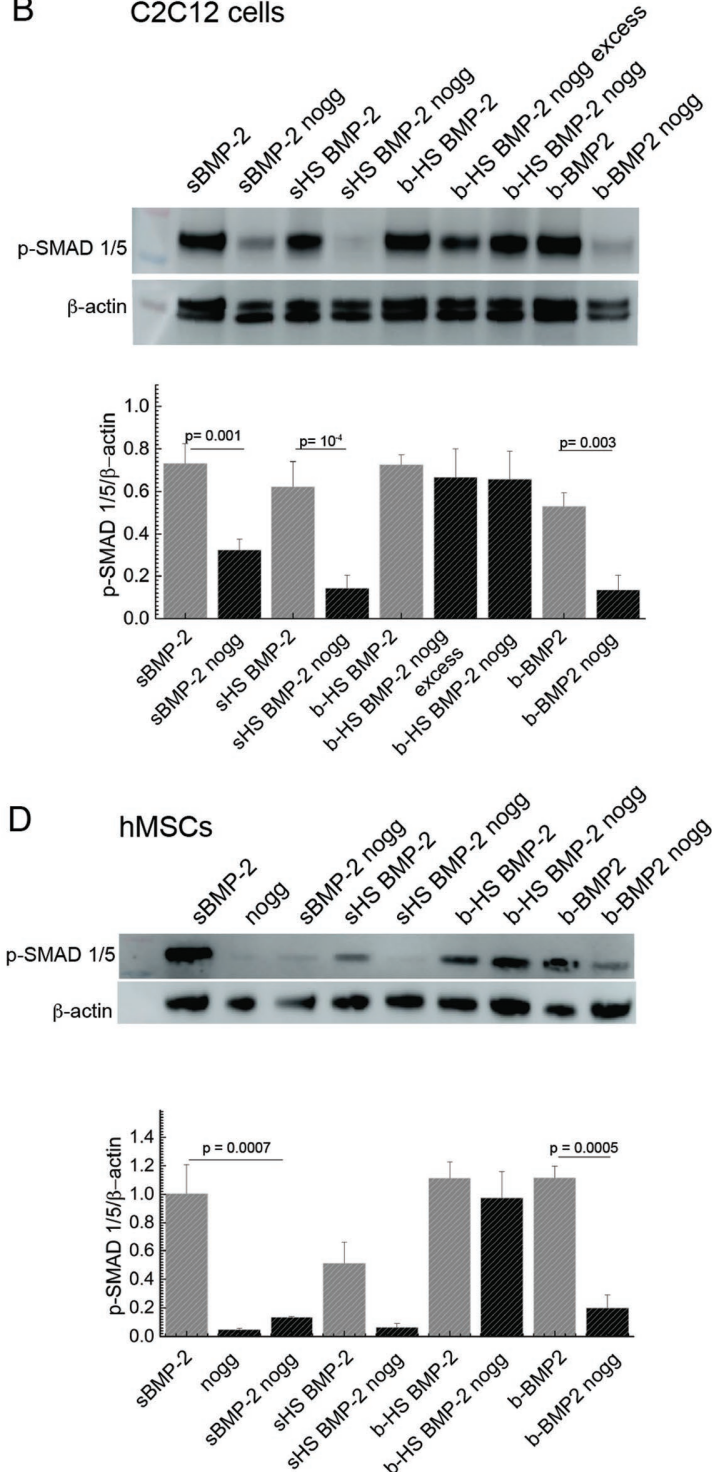

Figure 3. $A, B) C 2 C 12$ cells and $C, D) h M S C$ s plated on functionalized surfaces and lysed, at different time points $A, C)$ or after 90 min on substrates incubated $B, D$ ) with or without noggin. P-SMAD 1/5 expression analyzed by Western Blot.

marrow (hMSC) were plated for short periods (30-180 min) on the biomimetic platforms to compare the bioactivity of soluble BMP-2 (sBMP-2), immobilized b-BMP-2 and b-HS/BMP-2.

We first characterized the signaling response in $\mathrm{C} 2 \mathrm{C} 12$ cells, which form myotubes upon reaching confluency by switching to low serum condition, ${ }^{[32]}$ but in presence of BMP-2 their myogenic differentiation is inhibited resulting in transdifferentiation toward the osteogenic lineage. ${ }^{[33]}$ The phosphorylation level of SMAD 1/5 proteins, which are direct downstream effectors of the canonical BMP-SMAD signaling pathway, was used as indicator for BMP-2 bioactivity. We previously demonstrated that the covalent immobilization of BMP-2 on a surface via a heterobifunctional chemical linker retained the growth factor's biological activity and triggered BMP-mediated signaling in C2C12 cells. ${ }^{[34]}$ In the present study, we investigate the effect of BMP-2 surface presentation via b-HS, which resembles its presentation in the ECM, in comparison to its surface immobilization via biotin. In both platforms, BMP-2 surface concentration was determined

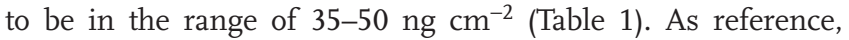
we used sBMP-2 at a concentration of $20 \times 10^{-9}$ M. $^{[2]}$ Surfaces presenting only b-HS were used as negative control. p-SMAD $1 / 5$ phosphorylation kinetics were determined at 30, 90, and 180 min after cell plating (Figure 3A,C). While SMAD 1/5 phosphorylation induced by sBMP-2 decreases during a $3 \mathrm{~h}$ stimulation period, the same could not be observed when BMP-2 is bound to b-HS or in the case of b-BMP2 immobilized on SAv. In particular, for the latter, SMAD $1 / 5$ phosphorylation peak is delayed at $90 \mathrm{~min}$ and remained stable also for $180 \mathrm{~min}$, in line with what has been previously observed for covalently immobilized BMP-2. ${ }^{[34]}$ After 180 min, the levels of phosphorylated 
SMAD $1 / 5$ are significantly higher in cells exposed to BMP-2 bound to b-HS than in presence of sBMP-2. Interestingly, for all the measured time points, a significant enhancement of p-SMAD 1/5 levels is observed when BMP-2 is presented through b-HS in comparison to b-BMP-2 (Figure 3A). To discard the possibility that $\mathrm{b}-\mathrm{HS}$ enhances SMAD phosphorylation independently of sBMP-2, we used the mutated form of BMP-2 unable to bind HS (EHBMP-2) on b-HS platforms. In this setting, SMAD 1/5 phosphorylation levels at $180 \mathrm{~min}$ were similar for both SBMP-2 and SEHBMP-2, and in the case of EHBMP-2 they were also not increased by the presence of b-HS (Figure S6, Supporting Information), suggesting that the enhancement of BMP-2 bioactivity is due to the specific presentation of BMP-2 by b-HS. It is thus conceivable that b-HS presents BMP-2 in the correct orientation to the BMP receptor complex.

Taken together, these results show that both immobilization strategies (b-BMP2 and b-HS/BMP-2) prolong the biological activity of the growth factor in comparison to its presentation to cells when added in the culture media. On b-HS presenting surfaces, the retention of BMP-2 might be favored by the cross-linking of the b-HS film (Figure S2, Supporting Information). We further demonstrate that the presentation via b-HS enhances BMP-mediated signaling in C2C12 cells in comparison to its direct immobilization on SAv. To test the role of b-HS as cofactor and to better elucidate the nature of the binding between b-HS and BMP-2, the affinity of the complex b-HS/BMP-2 to BMP receptors and the functional blocking of HSPGs should be addressed in future studies.

We observed a similar result in the kinetics of SMAD $1 / 5$ phosphorylation in primary hMSCs in response to the same immobilization strategy (Figure 3C). Indeed, on b-BMP2, SMAD $1 / 5$ phosphorylation is prolonged for $180 \mathrm{~min}$, while it decreased over time in presence of sBMP-2. In contrast to $\mathrm{C} 2 \mathrm{C} 12$ cells, the presence of b-HS does not enhance BMP-2 signaling in hMSCs in comparison to the presentation of BMP-2 immobilized on SAv, which generates also high and sustained levels of p-SMAD 1/5 expression. Furthermore, a short-time stimulation of hMSCs on b-HS/BMP-2 and b-BMP2 presenting surfaces is sufficient to promote osteogenic differentiation. Cells plated for 90 min on the biomimetic platforms in the presence of BMP-2, either immobilized or added to the cell media, and then replated for $24 \mathrm{~d}$ on untreated tissue culture plates, express alkaline phosphatase (ALP), a marker of osteogenic differentiation, ${ }^{[1]}$ without any additional factors (Figure S7, Supporting Information). The observed ALP expression in cells exposed to surfaces presenting b-HS and BMP-2 is significantly higher than the expression in cells exposed to sBMP-2. We conclude that early events triggered by the presentation of BMP-2 via b-HS are sufficient to activate the SMAD1/5 downstream signaling, which induces hMSCs differentiation towards the osteogenic lineage.

To assess whether the presence of b-HS prevents BMP-2 recognition by its antagonist noggin, as previously suggested for soluble HS (sHS), ${ }^{[20]}$ we analyze short-term BMP-2 signaling on surfaces presenting BMP-2 bound to b-HS or b-BMP2 bound to $\mathrm{SAv}$ in the presence of a double molar excess of noggin in solution (Figure 3B,D). As comparison, the same molar ratio has been used for sBMP-2 and for sBMP-2 bound to sHS. C2C12 and hMSCs responded in a comparable manner, demonstrating that noggin, by occupying to BMPRI and BMPRII binding epitopes, is able to inhibit the bioactivity of sBMP-2, sBMP-2 bound to sHS and b-BMP2 grafted on SAv. Surprisingly, when BMP-2 is bound to b-HS, the effect of noggin is negligible. To rule out the effect of BMP-2 released from b-HS (Figure S3, Supporting Information), we exposed cells to an excess of soluble noggin, which we expect to antagonize the biological activity of the released BMP-2. We demonstrate that even in presence of an excess of noggin in solution (ref. b-HS BMP-2 noggin excess), SMAD 1/5 phosphorylation levels remain high.

We speculate therefore that noggin does not efficiently recognize BMP-2 bound to b-HS, thus leaving a sufficient amount of BMP-2, which is bound to b-HS and still accessible to BMPRI and BMPRII to activate the SMAD 1/5 pathway. Future studies on the competition between b-HS/BMP-2 complex, noggin and BMP receptors might better clarify the impact of HS on BMP-2 interaction with noggin and with BMP receptors. Moreover, to consolidate our observations, platforms presenting controlled HS sulfation patterns and HS from different sources, are in the scope of future studies.

We designed a biomimetic and versatile platform for molecular and cellular studies, which presents immobilized BMP-2 alone (b-BMP2) or bound to b-HS grafted via its reducing end to SAv monolayers, similar to BMP-2 presentation by HSPGs in the ECM. By controlling the surface density and the stoichiometry of all components, we defined the apparent binding affinity between BMP-2 and b-HS and we demonstrated that BMP-2 can cross-link b-HS chains likely due to several independent $\mathrm{Hp} / \mathrm{HS}$ binding sites at the N-terminus. These platforms represent therefore a versatile and tunable biomimetic tool able to be exploited as active substrates for $\mathrm{C} 2 \mathrm{C} 12$ and hMSCs stimulation toward osteogenic differentiation. We show that (i) surface immobilization of BMP-2 prolongs the p-SMAD $1 / 5$ signaling activation with respect to the soluble BMP-2, (ii) the specific presentation via b-HS enhances the p-SMAD 1/5 levels on $\mathrm{C} 2 \mathrm{C} 12$ cells and prevents the antagonistic effect of noggin on both $\mathrm{C} 2 \mathrm{C} 12$ and hMSCs. Our study, therefore, highlights the potential importance of ECM HSPGs as regulators of BMP-2 activity, giving new insights into the molecular basis of ECM-BMP interactions and opening avenues for novel strategies to design biomimetic materials functionalized with BMP-2 in regenerative medicine.

\section{Experimental Section}

Further information on the experimental materials and methods are available in the Supporting Information.

\section{Supporting Information}

Supporting Information is available from the Wiley Online Library or from the author.

\section{Acknowledgements}

The authors thank Prof. Joachim Spatz (Department of Biophysical Chemistry, Heidelberg University and Max Planck Institute for Medical 
Research, Heidelberg) for his kind support and fruitful discussions, Dr. Hugues Lortat-Jacob (IBS, Grenoble, France) for providing HS and valuable feedback on the experiments; Prof. Walter Sebald, (Würzburg University, Germany) for providing EHBMP-2; Aïseta Baradji (University of Liverpool, UK and CICbiomaGUNE, San Sebastian, Spain) for helping with the SUV preparation; Dr. Burcu Minsky (Max Planck Institute for Intelligent Systems, Stuttgart) for support on SE measurements and, of course, the Cell Adhesion and signaling group (Heidelberg University). This project has received funding from European Union's Framework Program for Research and Innovation Horizon 2020 (20142020) under the Marie Sklodowska-Curie Grant Agreement No. H2020MSCA-IF-2014 and from CellNetworks Cluster, Heidelberg University, under the CellNetworks Postdoctoral Program 2014-2015. E.A.C.-A. greatly acknowledges the support of DFG (SFB TRR 79 projects M9 and B5). E.M., S.V.W., and E.A.C.-A. thank the Max Planck Society for support. R.P.R. acknowledges the Spanish Ministry for economy and competitiveness (project MAT2014-54867-R). P.K. has received funding from DFG Forschergruppe FOG 2165. P.K. and C.H. greatly acknowledge support by BSRT Berlin school for Regenerative Therapies.

Received: December 13, 2016 Published online:

[1] H. M. Ryoo, M. H. Lee, Y. J. Kim, Gene 2006, 366, 51.

[2] T. Katagiri, A. Yamaguchi, M. Komaki, E. Abe, N. Takahashi, T. Ikeda, V. Rosen, J. M. Wozney, A. Fujisawa-Sehara, T. Suda, J. Cell Biol. 1994, 127, 1755.

[3] A. Asakura, M. Komaki, M. Rudnicki, Differentiation 2001, 68, 245.

[4] F. Liu, A. Hata, J. C. Baker, J. Doody, J. Carcamo, R. M. Harland, J. Massague, Nature 1996, 381, 620.

[5] M. Geiger, R. H. Li, W. Friess, Adv. Drug Delivery Rev. 2003. 55, 1613.

[6] B. Sedaghati, B. Hoyer, A. Aigner, M. C. Hacker, M. Schulz-Siegmund, Regenerative Medicine - from Protocol to Patient: 3. Tissue Engineering, Biomaterial and Nanotechnology, Vol. 3, Springer, Cham, Switzerland 2016.

[7] S. Ahmed, R. P. Metpally, S. Sangadala, B. V. Reddy, J. Mol. Graphics Modell. 2010, 28, 670.

[8] E. Migliorini, A. Valat, C. Picart, E. A. Cavalcanti-Adam, Cytokine Growth Factor Rev. 2016, 27, 43.

[9] M. M. Martino, J. A. Hubbell, FASEB J. 2010, 24, 4711.

[10] X. Jiao, P. C. Billings, M. P. O'Connell, F. S. Kaplan, E. M. Shore, D. L. Glaser, J. Biol. Chem. 2007, 282, 1080.

[11] D. S. Bramono, S. Murali, B. Rai, L. Ling, W. T. Poh, Z. X. Lim, G. S. Stein, V. Nurcombe, A. J. van Wijnen, S. M. Cool, Bone 2012, 50, 954.

[12] R. Ruppert, E. Hoffmann, W. Sebald, Eur. J. Biochem. 1996, 237, 295.

[13] I. Matsuo, C. Kimura-Yoshida, Philos. Trans. R. Soc. Lond. B Biol. Sci. 2014, 369, 20130545

[14] a) M. C. Fisher, Y. Li, M. R. Seghatoleslami, C. N. Dealy, R. A. Kosher, Matrix Biol. 2006, 25, 27; b) J. Huegel, M. Enomoto-Iwamoto, F. Sgariglia, E. Koyama, M. Pacifici, Am. J. Pathol. 2015, 185, 1676.

[15] W. J. Kuo, M. A. Digman, A. D. Lander, Mol. Biol. Cell 2010, 21, 4028.

[16] B. L. Viviano, S. Paine-Saunders, N. Gasiunas, J. Gallagher, S. Saunders, J. Biol. Chem. 2004, 279, 5604.

[17] J. Groppe, J. Greenwald, E. Wiater, J. Rodriguez-Leon, A. N. Economides, W. Kwiatkowski, M. Affolter, W. W. Vale, J. C. Izpisua Belmonte, S. Choe, Nature 2002, 420, 636.

[18] a) G. Sanchez-Duffhues, C. Hiepen, P. Knaus, P. Ten Dijke, Bone 2015, 80, 43; b) Y. Matsumoto, K. Matsumoto, F. Irie, J. Fukushi, W. B. Stallcup, Y. Yamaguchi, J. Biol. Chem. 2010, 285, 19227.
[19] R. Gomes, C. Kirn-Safran, M. C. Farach-Carson, D. D. Carson, J. Musculoskeletal Neuronal Interact. 2002, 2, 511.

[20] S. Murali, B. Rai, C. Dombrowski, J. L. Lee, Z. X. Lim, D. S. Bramono, L. Ling, T. Bell, S. Hinkley, S. S. Nathan, J. H. Hui, H. K. Wong, V. Nurcombe, S. M. Cool, Biomaterials 2013, 34, 5594.

[21] S. Sarrazin, W. C. Lamanna, J. D. Esko, Cold Spring Harbor Perspect. Biol. 2011, 3, a004952.

[22] D. Thakar, E. Migliorini, L. Coche-Guerente, R. Sadir, H. Lortat-Jacob, D. Boturyn, O. Renaudet, P. Labbe, R. P. Richter, Chem. Commun. 2014, 50, 15148.

[23] E. Migliorini, D. Thakar, R. Sadir, T. Pleiner, F. Baleux, H. Lortat-Jacob, L. Coche-Guerente, R. P. Richter, Biomaterials 2014, 35, 8903.

[24] W. A. Hendrickson, A. Pahler, J. L. Smith, Y. Satow, E. A. Merritt, R. P. Phizackerley, Proc. Natl. Acad. Sci. USA 1989, 86, 2190.

[25] E. Migliorini, D. Thakar, J. Kuhnle, R. Sadir, D. P. Dyer, Y. Li, C. Sun, B. F. Volkman, T. M. Handel, L. Coche-Guerente, D. G. Fernig, H. Lortat-Jacob, R. P. Richter, Open Biol. 2015, 5, 150046.

[26] N. S. Gandhi, R. L. Mancera, Biochim. Biophys. Acta, 2012, 1824, 1374

[27] J. T. Gallagher, J. E. Turnbull, M. Lyon, Int. J. Biochem. 1992, 24, 553.

[28] C. I. Gama, S. E. Tully, N. Sotogaku, P. M. Clark, M. Rawat, N. Vaidehi, W. A. Goddard 3rd, A. Nishi, L. C. Hsieh-Wilson, Nat. Chem. Biol. 2006, 2, 467.

[29] a) N. Jastrebova, M. Vanwildemeersch, U. Lindahl, D. Spillmann, J. Biol. Chem. 2010, 285, 26842; b) M. Maccarana, Y. Sakura, A. Tawada, K. Yoshida, U. Lindahl, J. Biol. Chem. 1996, 271, 17804.

[30] L. Luca, M. A. Capelle, G. Machaidze, T. Arvinte, O. Jordan, R. Gurny, Int. J. Pharm. 2010, 391, 48.

[31] S. Masuda, K. Namba, H. Mutai, S. Usui, Y. Miyanaga, H. Kaneko, T. Matsunaga, Biochem. Biophys. Res. Commun. 2014, 447, 496.

[32] D. Yaffe, O. Saxel, Nature 1977, 270, 725.

[33] T. Katagiri, S. Akiyama, M. Namiki, M. Komaki, A. Yamaguchi, V. Rosen, J. M. Wozney, A. Fujisawa-Sehara, T. Suda, Exp. Cell Res. 1997, 230, 342.

[34] E. H. Schwab, T. L. Pohl, T. Haraszti, G. K. Schwaerzer, C. Hiepen, J. P. Spatz, P. Knaus, E. A. Cavalcanti-Adam, Nano Lett. 2015, 15, 1526.

[35] a) T. L. Pohl, J. H. Boergermann, G. K. Schwaerzer, P. Knaus, E. A. Cavalcanti-Adam, Acta Biomater. 2012, 8, 772; b) P. Paarmann, G. Dorpholz, J. Fiebig, A. R. Amsalem, M. Ehrlich, Y. I. Henis, T. Muller, P. Knaus, Int. J. Biochem. Cell Biol. 2016, 76, 51; c) T. Takada, T. Katagiri, M. Ifuku, N. Morimura, M. Kobayashi, K. Hasegawa, A. Ogamo, R. Kamijo, J. Biol. Chem. 2003, 278, 43229.

[36] B. Mulloy, C. Gee, S. F. Wheeler, R. Wait, E. Gray, T. W. Barrowcliffe, Thromb. Haemostasis 1997, 77, 668.

[37] R. Richter, A. Mukhopadhyay, A. Brisson, Biophys. J. 2003, 85, 3035.

[38] N. B. Eisele, S. Frey, J. Piehler, D. Gorlich, R. P. Richter, EMBO Rep. $2010,11,366$

[39] I. Carton, A. R. Brisson, R. P. Richter, Anal. Chem. 2010, 82, 9275.

[40] G. V. Dubacheva, T. Curk, B. M. Mognetti, R. Auzely-Velty, D. Frenkel, R. P. Richter, J. Am. Chem. Soc. 2014, 136, 1722.

[41] M. B. Huglin, J. Appl. Polym. Sci. 1965, 9, 1097.

[42] R. P. Richter, K. B. Rodenhausen, N. B. Eisele, M. Schubert, Coupling Spectroscopic Ellipsometry and Quartz Crystal Microbalance to Study Organic Films at the Solid-Liquid Interface, Vol. 52, Springer, Heidelberg 2014.

[43] a) V. Hagel, T. Haraszti, H. Boehm, Biointerphases 2013, 8, 36; b) T. T. Tsay, K. A. Jacobson, Biophys. J. 1991, 60, 360.

[44] W. Wagner, P. Horn, M. Castoldi, A. Diehlmann, S. Bork, R. Saffrich, V. Benes, J. Blake, S. Pfister, V. Eckstein, A. D. Ho, PLoS One 2008, 3, e2213.

[45] J. Kopf, P. Paarmann, C. Hiepen, D. Horbelt, P. Knaus, BioFactors 2014, 40, 171 . 